\title{
INFECÇÃO DO SÍTIO CIRÚRGICO EM PACIENTES SUBMETIDOS A CIRURGIAS CARDÍACAS: UMA ANÁLISE DO PERFIL EPIDEMIOLÓGICO
}

\section{SURGICAL SITE INFECTION IN PATIENTS SUBMITTED TO CARDIAC SURGERIES: AN EPIDEMIOLOGICAL PROFILE ANALYSIS}

\section{INFECCIÓN DEL SITIO CIRÚRGICO EN PACIENTES SOMETIDOS A CIRUGÍAS CARDÍACAS: ANÁLISIS DEL PERFIL EPIDEMIOLÓGICO}

Nelma de Jesus Braz ${ }^{1}$, Silma de Souza Evangelista ${ }^{2}$, Sintia Souza Evangelista ${ }^{1}$, Juliana Ladeira Garbaccio ${ }^{3}$, Adriana Cristina de Oliveira ${ }^{4}$.

\section{RESUMO}

Objetivo: Descrever a ocorrência da infecção do sítio cirúrgico em pacientes submetidos à revascularização do miocárdio e/ou implante de valva cardíaca e seu perfil epidemiológico. Método: Tratou-se de estudo retrospectivo, realizado em hospital de grande porte. Os dados foram coletados nos prontuários dos pacientes de cirurgias entre 2011 e 2015 . Realizou-se estatística descritiva e análise bivariada pelo Epi-info 6.4. Resultados: Foram revisados 280 prontuários de pacientes com idade média de 58 anos. As principais comorbidades encontradas nesses pacientes foram hipertensão arterial e dislipidemia, sendo comum ainda a febre reumática naqueles com implante de prótese valvar. Foram diagnosticadas 52 infecções do sítio cirúrgico, sendo 32 (61,5\%) durante a internação e 20 (38,5\%) por reinternação. As infecções incisionais superficiais foram prevalentes seguidas de órgão/cavidade. A idade, tempo de internação total e no pós-operatório e dias no Centro de Terapia Intensiva após a cirurgia foram associadas à ocorrência infecciosa em órgão/cavidade. O tempo médio de internação foi de 18,1 dias nos pacientes sem infecção e 25,4 naqueles com infecção. Conclusão: É imprescindível investir em medidas de prevenção de infecções que envolvem equipes assistenciais, profissionais de controle de infecção e núcleos de segurança na busca de melhores práticas assistenciais.

Descritores: Infecção; Infecção da ferida cirúrgica; Enfermagem; Vigilância em saúde pública; Vigilância epidemiológica.

\section{ABSTRACT}

Objective: The study aimed to describe the occurrence of surgical site infection in patients submitted to myocardial revascularization and/or cardiac valve implantation as well as its epidemiological profile. Method: This was a retrospective study performed at a large hospital. Data were collected from patients' records of surgeries performed from 2011 to 2015 . Descriptive statistics and bivariate analysis were performed using Epi-info 6.4. Results: We reviewed 280 patient records with a mean age of 58 years old. The main comorbidities found in those patients were arterial hypertension and dyslipidemia and, in those patients with valve prosthesis, rheumatic fever was common. Fifty-two (52) surgical site infections were diagnosed, 32 (61.5\%) happening during the hospitalization and 20 (38.5\%) in re-hospitalization. The superficial incisional infections were prevalent followed by organ/cavity. Age, total and post-operative hospitalization time, and days at the Intensive Care Center Unit after surgery were associated to infectious occurrence in organ/cavity. The mean time of hospitalization was 18.1 days in patients without infection and 25.4 in those with infection. Conclusion: It is imperative to invest in infection prevention measures involving care teams, infection control professionals and safety centers in the search for better care practices.

Descriptors: Infection; Surgical wound infection; Nursing; Public health surveillance; Epidemiological surveillance.

\section{RESUMEN}

Objetivo: Describir la ocurrencia de infección del sitio quirúrgico en pacientes sometidos a revascularización del miocardio, y/o implantación de valvas y su perfil epidemiológico. Método: Fue un estudio retrospectivo en un hospital grande. Los datos fueron recopilados de las historias clínicas de los pacientes, para las cirugías realizadas en los años 2011 a 2015. Las estadísticas descriptivas y análisis bivariados fueron realizados con Epi-Info 6.4. Resultados: Revisamos 280 prontuarios de los pacientes con edad media de 58 años. Las principales comorbilidades fueron la hipertensión y la dislipidemia, siendo común la fiebre reumática en las de implantación de prótesis valvular. Fueron diagnosticadas 52 infecciones de sitio quirúrgico, el 61,5\% durante la hospitalización y $38,5 \%$ en rehospitalización. Las infecciones incisionales superficiales más frecuentes, seguidas por órganos/cavidad. La edad, tiempo de internación total y en el postoperatorio y días en el Centro de Terapia Intensiva, después de la cirugía fueron asociadas a la ocurrencia infecciosa en órgano/cavidad. La duración media de internación en pacientes sin infección fue de 18,1 días, y con infección fue 25,4 días. Conclusión: Es imprescindible investir en medidas de prevención con participación de los equipos de asistencia, profesionales de control de infección y núcleos de seguridad buscando mejores prácticas asistenciales. Descriptores: Infección; Infección de la herida quirúrgica; Enfermería; Vigilancia en salud pública; Vigilancia epidemiológica.

${ }^{1}$ Graduada em Enfermagem. Mestre em Promoção da Saúde e Prevenção de Agravos pela Universidade Federal de Minas Gerais. ${ }^{2}$ Graduada em Estatística. Mestre em Estatística pela Universidade Federal de Minas Gerais. Docente na Universidade Federal de Santa Catarina. ${ }^{3}$ Graduada em Enfermagem e Odontologia. Doutora em Enfermagem pela Universidade Federal de Minas Gerais. Docente na Pontifícia Universidade Católica de Minas Gerais. ${ }^{4}$ Graduada em Enfermagem. Pós-Doutora pela New York University. Docente na Universidade Federal de Minas Gerais.

Como citar este artigo:

Braz NJ, Evangelista SS, Evangelista SS, et al. Infecção do sítio cirúrgico em pacientes submetidos a cirurgias cardíacas: uma análise do perfil epidemiológico. Revista de Enfermagem do Centro-Oeste Mineiro. 2018;8:e1793. [Access in:__. DOI: http://dx.doi.org/10.19175/recom.v7i0.1793 


\section{INTRODUÇÃO}

As cirurgias cardíacas encontram-se entre as mais realizadas no mundo. A cada 1.000 .000 habitantes por ano, ocorrem em torno de 2000 cirurgias cardíacas nos Estados Unidos, 900 na Europa e 350 no Brasil ${ }^{(1)}$. As Cirurgias de Revascularização do Miocárdio (CRVM) e as de implantes de valvas cardíacas são os dois tipos mais comuns, podendo ser realizadas de forma separada ou concomitantes.

A CRVM constitui uma das modalidades terapêuticas do tratamento da Doença Arterial Coronariana que, em geral, desenvolve-se em pacientes que apresentam fatores predisponentes como tabagismo, histórico familiar, obesidade, hipertensão arterial sistêmica (HAS), diabetes mellitus, dislipidemia, dentre outras $^{(2)}$. Os implantes de valvas cardíacas, por sua vez, ocorrem para otimizar o estado de saúde dos portadores de valvopatias que se desenvolvem em decorrência da idade avançada ou complicações resultantes da febre reumática, que é responsável pela etiologia de $70 \%$ dos casos no Brasil, conferindo ao país um perfil epidemiológico particular em relação aos países desenvolvidos. As valvopatias mais comuns na doença reumática são a estenose e a insuficiência mitral, seguida da aórtica que acometem pacientes jovens, sendo, na senilidade, a estenose aórtica por calcificações mais encontrada e quase sempre associada à dislipidemia, ao tabagismo e à $\mathrm{HAS}^{(3)}$.

As Infecções de sítio cirúrgico (ISC) representam a complicação mais comum entre pacientes submetidos a procedimentos cirúrgicos, destacando-se entre as principais Infecções Relacionadas a Assistência à Saúde (IRAS). Em hospitais norte-americanos, no ano de 2011, $21,8 \%$ dos pacientes hospitalizados acometidos por IRAS desenvolveram ISC, sendo essa a segunda principal causa dessas infecções ${ }^{(4)}$. No Brasil, as ISC ocupam a terceira posição entre todas as IRAS, com taxas que variam entre $14 \%$ e $16 \%$ entre pacientes hospitalizados ${ }^{(5)}$.

Entre as cirurgias cardíacas, as ISC são graves e de grande impacto econômico em decorrência do prolongamento de tempo de internação (entre 15 a 45 dias), do custo (seis vezes maior, considerando o paciente sem infecção, variando de $\$ 19.000,00$ até $\$ 150.000,00)$ e, por elevar a taxa de mortalidade (em cerca de $8 \%$ a $20 \%$ ), mesmo após o tratamento ${ }^{(6-8)}$.
O desfecho infeccioso pós-cirúrgico é favorecido por uma multiplicidade de fatores inter-relacionados que abrangem diversos aspectos assistenciais do pré, trans e pósoperatório, além de envolver múltiplos fatores organizacionais e humanos, exigindo a atuação de uma equipe multiprofissional treinada e capacitada na prevenção desses eventos. Estudos demonstram que o enfermeiro tem exercido papel chave na implantação e sustentação de medidas de vigilância aos fatores de risco e na prevenção da ISC, como a implantação de bundles e da lista de verificação cirúrgica, por exemplo, ferramentas que têm contribuído para a redução significativa de ISC em diversas instituições ${ }^{(9-10)}$.

No entanto, os fatores de risco definidos podem não se comportar da mesma forma como preditores da infecção, em pacientes submetidos à cirurgia cardíaca, em diferentes populações. Sob essa ótica e, dada a relevância da ISC em cirurgias cardíacas, o estudo de populações diferenciadas deve ser conduzido a fim de que se possa estabelecer se os estudos encontram em consonância com aqueles descritos em literatura ou se podem ser identificados comportamentos epidemiológicos diferenciados em distintas populações.

Diante do exposto, o presente estudo tem como objetivo descrever a ocorrência de ISC em pacientes submetidos à CRVM e/ou implante de valva cardíaca e seu perfil epidemiológico.

\section{MÉTODOS}

Tratou-se de um estudo retrospectivo conduzido em hospital universitário, público de Minas Gerais, com 509 leitos registrados no Cadastro Nacional de Estabelecimentos de Saúde, para atendimento exclusivo a pacientes do Sistema Único de Saúde (SUS).

Foram elegíveis para o estudo, prontuários de pacientes submetidos à CRVM ou implante de valva cardíaca ou ambas, de forma concomitante, no período de 01 de janeiro de 2011 a 31 de dezembro de 2015. Excluem-se aqueles prontuários cujos pacientes faleceram em período igual ou inferior a 24 horas após o procedimento, ou que não se submeteram a cirurgias na instituição do estudo, totalizando 280 prontuários analisados.

A coleta de dados ocorreu por meio da revisão de prontuários, adotando-se um instrumento que contemplava dados sociodemográficos, da internação, do procedimento cirúrgico, além de aspectos 
relacionados à ocorrência de ISC nesses pacientes.

As ISC foram classificadas em incisional superficial, incisional profunda e órgão/cavidade, conforme critérios de notificação epidemiológica do National Healthcare Surveillance Network do Centers for Disease Control (NHSN-CDC) ${ }^{(11)}$ versão 2015, baseados na estrutura acometida, no tempo de ocorrência e na presença de sinais e sintomas.

Os dados foram compilados num banco de dados no Programa EPi-info, versão 6.04, analisados por estatística descritiva, medidas de tendência central (média e mediana) e de dispersão (desvio-padrão). O teste de MannWhitney foi utilizado na análise bivariada, na verificação da relação entre as variáveis propostas e a ocorrência de ISC. Foram consideradas significativas, variáveis que apresentaram $p<0,05$.

O estudo teve aprovação do Comitê de Ética em Pesquisa (COEP), sob o parecer CAAE53843316.4.0000.5149, respeitando as regulamentações descritas na Resolução $n^{\circ}$ 466/2012, do Conselho Nacional de Saúde (CNS). Por se tratar de um estudo com coleta de dados de fontes secundárias, contidas em prontuário, houve dispensa do Termo de Consentimento Livre e Esclarecido (TCLE) por parte do COEP.

\section{RESULTADOS E DISCUSSÃO}

Foram identificados 280 prontuários de pacientes submetidos a cirurgias cardíacas no período analisado, sendo 152 (54,3\%) à CRVM, $105(37,5 \%)$ à implante de prótese valvar e 23 $(8,2 \%)$ a ambas as cirurgias, de forma concomitante.

Predominou, na amostra geral e por tipo de cirurgia, pacientes do sexo masculino 156 $(55,7 \%)$, casados $165(65,7 \%)$, diferindo para o sexo feminino $64(61,0 \%)$, aqueles submetidos a implante valvar cardíaco. $\mathrm{O}$ ano de maior realização de cirurgias foi em 2015 - 71 (25,4\%) e o menor em 2014 - 37 (13,2\%). A maioria dos pacientes, 252 (90\%) era procedente do domicílio. Em todos os anos, os procedimentos cirúrgicos combinados ocorreram em quantidade inferior aos demais, isoladamente. As CRVM foram prevalentes, exceto em 2015, em que predominou o implante de prótese valvar (Tabela 1).

Tabela 1 - Características sociodemográficas dos pacientes $(n=280)$ por tipo de cirurgia realizada, no período de 2011 a 2015, Belo Horizonte, 2017.

\begin{tabular}{|c|c|c|c|c|c|c|c|c|}
\hline \multirow{3}{*}{ Características } & \multicolumn{6}{|c|}{ Tipo de cirurgia realizada } & & \\
\hline & \multicolumn{2}{|c|}{ CRVM } & \multicolumn{2}{|c|}{$\begin{array}{l}\text { Implante de valva } \\
\text { cardíaca }\end{array}$} & \multicolumn{2}{|c|}{$\begin{array}{l}\text { CRVM e implante } \\
\text { de valva cardíaca }\end{array}$} & \multicolumn{2}{|c|}{ Total } \\
\hline & $\mathrm{N}$ & $\%$ & $\mathrm{~N}$ & $\%$ & $\mathrm{~N}$ & $\%$ & $\mathrm{~N}$ & $\%$ \\
\hline \multicolumn{9}{|l|}{ Sexo } \\
\hline Feminino & 52 & 34,2 & 64 & 61,0 & 8 & 34,8 & 124 & 44,3 \\
\hline Masculino & 100 & 65,8 & 41 & 39,0 & 15 & 65,2 & 156 & 55,7 \\
\hline \multicolumn{9}{|l|}{ Estado Civil ( $n=251)$} \\
\hline Solteiro & 18 & 13,2 & 33 & 34,7 & 2 & 10,5 & 53 & 21,1 \\
\hline Casado & 97 & 70,8 & 53 & 55,8 & 15 & 79,0 & 165 & 65,7 \\
\hline Viúvo & 15 & 10,9 & 8 & 8,4 & 2 & 10,5 & 25 & 10,0 \\
\hline Divorciado & 7 & 5,1 & 1 & 1,1 & 0 & 0,0 & 8 & 3,2 \\
\hline Sem informação & 15 & - & 10 & - & 4 & - & 29 & - \\
\hline \multicolumn{9}{|l|}{ Procedência } \\
\hline Comunidade & 135 & 88,8 & 95 & 90,5 & 22 & 95,7 & 252 & 90 \\
\hline Hospitalar & 17 & 11,2 & 10 & 9,5 & 1 & 4,3 & 28 & 10 \\
\hline \multicolumn{9}{|l|}{ Ano de realização } \\
\hline 2011 & 45 & 29,6 & 17 & 16,2 & 2 & 8,7 & 64 & 22,9 \\
\hline 2012 & 32 & 21,1 & 16 & 15,2 & 5 & 21,7 & 53 & 18,9 \\
\hline 2013 & 27 & 17,8 & 22 & 21,0 & 6 & 26,1 & 55 & 19,6 \\
\hline 2014 & 18 & 11,8 & 18 & 17,1 & 1 & 4,3 & 37 & 13,2 \\
\hline 2015 & 30 & 19,7 & 32 & 30,5 & 9 & 39,1 & 71 & 25,4 \\
\hline
\end{tabular}

A idade média global dos pacientes avaliados foi de 58 anos (DP=13,7). Distribuídos por procedimento cirúrgico, observou-se que a idade média na CRVM foi de 62,7 anos (DP=10,1). 
Nos implantes de próteses, a idade foi de 51,1 anos $(D P=15,6)$ e, para as cirurgias realizadas concomitantes, foi de 63,6 anos ( $D P=11,4)$. A variação da idade média dos pacientes submetidos a procedimentos cirúrgicos cardíacos, entre 51 a 63 anos, está de acordo com a literatura nacional e internacional ${ }^{(8,12-16)}$.

As comorbidades mais frequentemente presentes entre os pacientes que se submeteram à CRVM foram a HAS em 37 (24,2\%) e a dislipidemia em 28 (18,3\%). Em relação ao implante de prótese valvar detectou-se que 20 $(19,6 \%)$ apresentavam febre reumática e 18 $(17,1 \%)$ eram hipertensos. Entre os pacientes submetidos às duas cirurgias, 5 (23,3\%) eram portadores de HAS e 3 (14\%) dislipidêmicos. Geralmente, na faixa etária em que se encontravam os pacientes deste estudo, é comum a presença de comorbidades potencias, que podem desencadear cardiopatias passíveis de tratamento cirúrgico, como foi o caso de HAS, febre reumática e dislipidemia. A idade, embora seja fator de risco para ISC, não é passível de modificação.

A média de internação prévia ao procedimento cirúrgico foi de 12,8 dias (DP=9,5). Por procedimento, registrou-se na CRVM, o tempo médio de 13,6 dias ( $D P=9,3$ ), em implantes de válvulas 11,9 dias ( $D P=10)$, e após ambas as cirurgias concomitantes 11 dias ( $D P=7,7)$.

Do total da amostra analisada, 105 pacientes $(37,5 \%)$ necessitaram internação prévia à cirurgia no $C T I$, em média de 4,7 dias $(D P=3,3)$. Pacientes submetidos à CRVM necessitaram de $4,8$ dias ( $D P=3,2)$, enquanto pacientes submetidos a implante de prótese cardíaca e à realização concomitante dos dois procedimentos necessitaram de 4,5 dias $(D P=4,1)$ e 3,5 dias $(D P=1,4)$ respectivamente.

Observou-se que $260(92,9 \%)$ pacientes submeteram-se às cirurgias de forma eletiva e 20 $(7,1 \%)$ a procedimentos cirúrgicos em caráter de urgência. Em $270(96,8 \%)$ pacientes, o potencial de contaminação das cirurgias foi limpo.

A avaliação do período transoperatório revelou que a circulação extracorpórea ocorreu em 278 (99,3\%). O tempo médio de circulação extracorpórea (CEC) foi de aproximadamente 82 minutos ( $D P=25,7)$ para $C R V M, 94,1$ minutos $(D P=32,3)$ em implantes de valvas cardíacas e $116,4$ minutos ( $D P=25,2)$ em pacientes submetidos aos dois procedimentos concomitantes. Para os três procedimentos, o tempo médio de isquemia durante a CEC foi de
$66,2$ minutos ( $D P=24,4), 75,9$ minutos ( $D P=27,3$ ) e $93,0$ minutos ( $D P=18,3)$, respectivamente. As CRVM realizadas isolada ou concomitantemente com o implante de válvula cardíaca ocorreram em $177(63,2 \%)$ pacientes.

Registrou-se a utilização de antimicrobianos profiláticos em 270 (96,4\%) pacientes enquanto $10(3,6 \%)$ iniciaram o uso de antibiótico terapêutico antes do procedimento cirúrgico para tratamento de quadro infeccioso. Nestes pacientes, a dose inicial foi administrada até uma hora antes da incisão em 218 (88,6\%) prontuários que continham essa informação e após o início da cirurgia em 28 (11,4\%). O intervalo médio entre a administração da primeira dose do antibiótico e o início da incisão cirúrgica foi de 45,1 minutos ( $D P=20,2$ ), estimado entre os 246 pacientes, para os quais esta informação estava disponível. Para serem efetivos, os antimicrobianos precisam ser administrados em até uma hora antes do procedimento, de maneira a garantir a concentração tissular e sérica ideal no momento da incisão, exceto em casos de uso da vancomicina e fluorquinolonas que devem ser administradas 120 minutos antes do início da cirurgia, devido ao tempo prolongado de infusão necessário para essas ${ }^{(5,14)}$. Nesse sentido, a maioria $(88,6 \%)$ dos antimicrobianos foi administrada no tempo adequado.

A duração da antibioticoprofilaxia em até 24 horas ocorreu em 188 (73,2\%) pacientes; duração entre 24 a 48 horas em $65(25,3 \%)$ e 4 $(1,6 \%)$ em período maior que 48 horas. 0 antibiótico de escolha foi a cefazolina em 243 $(90,0 \%)$ pacientes e a oxacilina em 19 (7,0\%). 0 tempo cirúrgico médio estimado foi de 247,7 minutos ( $D P=47,2)$ sendo que, em quatro prontuários, essa informação não foi registrada.

Para os pacientes submetidos às cirurgias cardíacas no período analisado, 52 (18,6\%) foram diagnosticadas com ISC, taxa essa bem superior às descritas na literatura que oscilam no mundo todo entre $1,6 \%$ e $9,4 \%^{(7-8,13-15,17)}$. Daqueles diagnosticados com ISC, 32 casos (61,5\%) ocorreram durante a internação e 20 (38,5\%) após a alta hospitalar. Destes, 12 (25,5\%) necessitaram readmissão para tratamento da ISC.

De acordo com a estrutura acometida, as infecções incisionais superficiais ocorreram em 34 $(65,4 \%)$ pacientes com ISC, seguida das infecções de órgão/cavidade em 12 (23,1\%) e incisional profunda em seis (11,5\%). Entre as infecções de órgão/cavidade, as mediastinites foram as mais 
prevalentes, com nove $(75 \%)$ casos, seguidas da endocardite, com dois $(16,7 \%)$. Similarmente aos demais estudos, as ISC incisionais superficiais são as mais diagnosticadas, tendo sido encontradas no presente estudo em $65 \%$ das notificações de ISC $^{(15-16,18)}$. Contudo, chama atenção a ocorrência de diversos casos de infecções de órgão/cavidade, mediastinites e endocardites, que são mais graves e induzem à reinternação, acarretando repercussões mais graves para os pacientes. Entre os procedimentos realizados, os casos de infecção se distribuíram da seguinte forma: $34(65,4 \%)$ ocorreram entre pacientes submetidos às CRVM; $12(23,1 \%)$ naqueles após implante de prótese; e seis $(11,5 \%)$ após realização de ambas as cirurgias concomitantes (Tabela 2).

Tabela 2 - Distribuição da infecção do sítio cirúrgico segundo estrutura acometida e momento do diagnóstico em pacientes submetidos à cirurgia de revascularização do miocárdio, implante de válvulas cardíacas e ambas, no período de 2011 a 2015. Belo Horizonte, 2017.

\begin{tabular}{|c|c|c|c|c|c|c|c|c|}
\hline \multirow{3}{*}{ Características das ISC } & \multicolumn{6}{|c|}{ Tipo de cirurgia } & & \\
\hline & \multicolumn{2}{|c|}{ CRVM } & \multicolumn{2}{|c|}{$\begin{array}{l}\text { Implante de válvula } \\
\text { cardíaca }\end{array}$} & \multicolumn{2}{|c|}{$\begin{array}{c}\text { CRVM e implante de } \\
\text { válvula }\end{array}$} & \multicolumn{2}{|c|}{ Total } \\
\hline & $\mathrm{N}$ & $\%$ & $\mathrm{~N}$ & $\%$ & $\mathrm{~N}$ & $\%$ & $\mathrm{~N}$ & $\%$ \\
\hline \multicolumn{9}{|l|}{ Sítio de infecção } \\
\hline Incisional superficial & 25 & 75,8 & 6 & 46,2 & 03 & 50,0 & 34 & 65,4 \\
\hline Incisional profunda & 3 & 9,1 & 2 & 15,4 & 1 & 17,7 & 6 & 11,5 \\
\hline Órgão/cavidade & 5 & 15,2 & 5 & 38,4 & 2 & 33,3 & 12 & 23,1 \\
\hline \multicolumn{9}{|l|}{ Momento do diagnóstico } \\
\hline Durante a internação & 18 & 54,5 & 10 & 76,9 & 4 & 66,7 & 32 & 61,5 \\
\hline Após a alta & 15 & 45,5 & 3 & 23,1 & 2 & 33,3 & 20 & 38,5 \\
\hline
\end{tabular}

O tempo médio global decorrido entre a cirurgia e o diagnóstico da ISC foi de 18,1 dias $(D P=10,2)$. A literatura corrobora os dados observados, apontando uma variação da manifestação entre $014^{\circ}$ e o $30^{\circ}$ dia da cirurgia $^{(15)}$. Por procedimentos, foi estimado em $17,6$ dias ( $D P=8,5)$ após CRVM; 15,0 dias ( $D P=5,4)$ após cirurgias de troca de válvula e 26,7 dias $(D P=20,2)$ em pacientes submetidos às duas cirurgias concomitantes. A média de dias entre a cirurgia e o diagnóstico de ISC foi de 12,8 dias $(D P=6,0), 30$ dias para pacientes internados e 26,3 dias $(D P=10,1)$ em pacientes após a alta. Embora a maioria das ISC, 32 (65\%), tenha sido diagnosticada durante a internação e 20 (38,5\%) tenham sido detectadas por dados de reinternação do paciente, esse valor é preocupante e pode estar sendo ainda subestimado, uma vez que a vigilância pós-alta pode não ter sido realizada e, além disso, alguns pacientes podem ter desenvolvidos sinais e sintomas de ISC e não ter retornado ao hospital para serem diagnosticados e tratados adequadamente. Esse dado reforça a necessidade de proceder ao acompanhamento dos pacientes por um período após a alta ou até mesmo aprimorar o sistema interno de notificação de reinternações dos pacientes por complicações infecciosas, conforme preconizado pelo $\mathrm{CDC}^{(16)}$.

De modo geral, as taxas de ISC são influenciadas pela qualidade da vigilância, tempo de seguimento do paciente (principalmente no pós-alta), os métodos de coleta de dados, a organização do relatório de dados para os programas nacionais e para o feedback. Os valores encontrados têm sido frequentemente subestimados, principalmente quando se compara os dados institucionais ou nacionais de um programa de vigilância com dados de estudos bem delineados ${ }^{(19-21)}$.

No que se refere ao tempo médio de internação dos pacientes sem ISC, esse foi de 18,1 dias, enquanto os pacientes com ISC permaneceram uma semana a mais (25,4 dias). Esses achados estão compatíveis com outros estudos que apresentaram média de internação dos pacientes sem manifestação infecciosa de nove a 18 dias $^{(22)}$ e, na vigência da ISC, o prolongamento ultrapassando 45 dias. Esse tempo de internação pode ser até três vezes maiores em relação aos que não apresentaram ISC, contribuindo assim para o aumento dos 
custos assistenciais associados e para o desgaste do paciente envolvido e seus familiares ${ }^{(6-7,23-24)}$.

A média de internação dos pacientes após o diagnóstico da ISC foi de 34,4 dias (DP=19,0). O tempo médio de permanência hospitalar após o diagnóstico de ISC foi de 33,9 dias (DP=18,8) nos pacientes diagnosticados durante a internação. Destacou-se como maior tempo de internação hospitalar após o diagnóstico da ISC, pacientes submetidos implante de válvula cardíaca, média de 39,4 dias, seguidos da CRVM - 31,4 dias, com discreto aumento em relação àqueles submetidos às duas cirurgias de forma concomitante - 30,8 dias.

Naqueles pacientes diagnosticados após a alta e que necessitaram readmissão para tratamento da ISC, a média de permanência foi de 33,9 dias ( $D P=25,0$ ), sendo esta média de 25,4 naqueles submetidas à CRVM, 9,5 em pacientes submetidos à troca valvar e 55 , em pacientes submetidos aos dois procedimentos.

Pacientes sem ISC apresentaram média de 19 dias de internação hospitalar ( $D P=22,3)$, enquanto naqueles com infecção, a média foi de 25 dias ( $D P=19,2)$. A permanência no CTI em pacientes com ISC foi de 6 dias ( $D P=13,5)$, enquanto sem ISC foi de 4 dias (DP=13,9).

Da amostra total, 270 (96,4\%) pacientes evoluíram com alta domiciliar, nove $(3,2 \%)$ a óbito e um $(0,4 \%)$ foi transferido para outro hospital. A readmissão foi necessária em 69 $(25,5 \%)$ pacientes, sendo que destes, $12(17,4 \%)$ foram por ISC. Dos pacientes que faleceram, cinco $(55,6 \%)$ apresentaram outros tipos de IRAS, um evoluiu com ISC, um ISC e pneumonia e em apenas dois $(22,2 \%)$ não houve histórico infeccioso.

O desenvolvimento da ISC tem sido descrito como multifatorial, com o envolvimento de fatores de risco relacionados ao patógeno como a carga microbiana envolvida, sua patogenicidade e sua infectividade; ao paciente como os extremos de idade, doenças preexistentes, período de internação préoperatória, situação nutricional, descontrole glicêmico, tabagismo, obesidade, imunossupressão, infecções remanescentes e realização de transfusão perioperatória de hemoderivados; e ao procedimento cirúrgico: a remoção de pelos, preparo da área operatória, antissepsia das mãos do cirurgião, profilaxia antimicrobiana, técnica cirúrgica, assepsia, tempo prolongado de cirurgia, problemas na oxigenação e normotermia do paciente ${ }^{(25)}$. Na análise dos fatores determinantes da ISC foram analisados dados sociodemográficos e os momentos préoperatório, transoperatório e pós-operatório em relação aos pacientes com e sem ISC.

No presente estudo, dentre os fatores de risco relacionados ao paciente, a idade foi a única que apresentou relação com o desfecho ISC $(p=0,011)$, dentre as demais variáveis sociodemográficas analisadas (Tabela 3). Esse fato está em consonância com os demais estudos que apresentam extremos de idade como fator de risco para a ISC ${ }^{(12,16)}$.

Entre as variáveis analisadas no período pré-operatório, período de internação $(p=0,902)$, tempo no CTI pré-operatório $(p=0,253)$ e ocorrência da ISC, não se mostraram estatisticamente significativas. $\mathrm{O}$ período de internação pré-operatória prolongado, embora seja um fator de risco para a ISC, não foi associado a ISC neste estudo. Porém, o seu aumento no pós-operatório, mostrou-se significativamente associado ao desfecho ISC. O aumento do tempo de internação no pré ou pósoperatório favorece a aquisição da microbiota hospitalar pelo paciente, tornando maior o risco de colonização e o desenvolvimento de ISC ${ }^{(16)}$.

No transoperatório, as variáveis: a utilização de circulação extracorpórea $(p=0,831)$ e o tempo cirúrgico $(p=0,078)$ não apresentaram significância estatística, bem como o uso de antibiótico profilático $(p=0,280)$, intervalo de administração da primeira dose antes da incisão $(p=0,823)$, uso de doses adicionais durante a cirurgia $(p=1,00)$ e duração da profilaxia até 24 horas $(p=0,763)$.

Outro fator de risco para ISC, relacionados ao procedimento cirúrgico avaliado pelo estudo, foi o tempo de duração da cirurgia. De acordo com a literatura, quanto maior a duração da cirurgia, maior o risco de ISC devido à exposição e manipulação tecidual. Procedimentos prolongados são aqueles que duram mais do que $75 \%$ do tempo em outros semelhantes, ou seja, o percentil 75 de cada tipo de cirurgia ${ }^{(16)}$. Contudo, esse fator não esteve relacionado significativamente com o desfecho ISC.

Quanto aos fatores de risco relacionados ao procedimento cirúrgico citados acima, a antibioticoprofilaxia administrada em até uma hora antes da incisão foi relacionada como fator protetivo para o desenvolvimento de ISC. A administração de antibióticos profiláticos previamente à cirurgia objetiva e redução da carga microbiana na ferida operatória. Os 
procedimentos que demandam essa prática são as cirurgias consideradas potencialmente contaminadas e algumas cirurgias limpas, cujas consequências de uma ISC podem ser severas, como as cirurgias cardíacas abertas (como é o caso deste estudo, em que $96,4 \%$ dos pacientes receberam a profilaxia adequadamente), as substituições articulares, a prótese vascular e as craniotomias $^{(14,16)}$.

Tabela 3 - Características relacionadas à ocorrência da ISC entre os pacientes submetidos à cirurgia de revascularização do miocárdio, implante de válvulas cardíacas ou ambas as cirurgias $(n=280)$, no período de 2011 a 2015. Belo Horizonte, 2017.

\begin{tabular}{|c|c|c|c|c|c|c|c|}
\hline \multirow{3}{*}{ Características } & \multicolumn{6}{|c|}{ Infecção de sítio cirúrgico } & \multirow{3}{*}{ Valor-p } \\
\hline & \multicolumn{3}{|c|}{ Ausente } & \multicolumn{3}{|c|}{ Presente } & \\
\hline & Média & D.P. & Mediana & Média & D.P. & Mediana & \\
\hline Idade (anos) & 57,5 & 13,4 & 60,0 & 61,8 & 15,7 & 66,5 & $0,011^{1}$ \\
\hline Tempo de internação (dias) & 11,5 & 6,1 & 9,5 & 13,1 & 10,1 & 11,0 & $0,902^{1}$ \\
\hline Internação CTI (pré-operatório) & 5,4 & 3,6 & 4,5 & 4,5 & 3,2 & 3,0 & $0,253^{1}$ \\
\hline Tempo de CEC & 88,2 & 28,2 & 88,0 & 92,6 & 36,6 & 89,5 & $0,831^{1}$ \\
\hline $\begin{array}{l}\text { Intervalo da administração da } 1 \text { a dose do ATB antes } \\
\text { da incisão }\end{array}$ & 43,2 & 21,0 & 45,0 & 43,9 & 25,4 & 45,0 & $0,823^{1}$ \\
\hline Tempo cirúrgico & 244,9 & 56,2 & 240,0 & 260,1 & 59,8 & 255,0 & $0,078^{1}$ \\
\hline Tempo de internação após a cirurgia & 15,5 & 18,3 & 11,0 & 25,2 & 17,4 & 20,0 & $<0,001^{1}$ \\
\hline Dias no CTI após cirurgia & 7,2 & 13,9 & 6,0 & 11,9 & 13,5 & 6,0 & $<0,001^{1}$ \\
\hline Tempo total de internação & 23,6 & 22,3 & 19,0 & 32,5 & 19,4 & 25,0 & $<0,001^{1}$ \\
\hline
\end{tabular}

1: Mann-Whitney

De maneira geral, a profilaxia não deve exceder 24 horas, exceto em cirurgias cardiotorácicas, em que têm se aceitado a profilaxia até 48 horas após o procedimento, apesar de não ser uma prática embasada cientificamente ${ }^{(5)}$. No presente estudo, a maioria das profilaxias antimicrobianas foi mantida por 24 horas (73,2\% dos pacientes) e, em $25,3 \%$, por 24 a 48 horas, conforme preconizado na literatura.

Outras condições do pós-operatório que foram significativas estatisticamente em relação ao desfecho infeccioso cirúrgico, como demonstrado na Tabela 3, foram o tempo de internação após cirurgia $(p<0,001)$, dias no CTI após cirurgia $(p<0,001)$ e tempo total de internação hospitalar $(p<0,001)$. 0 prolongamento da internação na cirurgia cardíaca pode estender em até quatro vezes a permanência do paciente, os gastos em saúde em até seis vezes, impactando financeiramente a Instituição, a vida social do paciente e familiar ${ }^{(22-}$ 23). A permanência prolongada em ambiente hospitalar pode ainda favorecer o desfecho infeccioso pela maior probabilidade de contaminação neste ambiente $\mathrm{e}^{(4-5,17)}$.

\section{CONCLUSÃO}

No presente estudo, as variáveis idade, o tempo total de internação, o tempo de internação no pós-operatório e dias no $\mathrm{CTI}$, após a cirurgia, foram associados à ocorrência da ISC em CRVM e de implante de prótese valvar, sendo as três últimas passíveis de modificação, dependendo de cada caso.

As taxas de infecções foram elevadas nos três tipos de cirurgias cardíacas analisadas, sendo as ISC superficiais, as mais prevalentes, seguidas das ISC de órgão/cavidade. O tempo médio até o diagnóstico dessas ISC foi elevado, sendo considerável o número de infecções detectadas por meio da reinternação hospitalar. Demonstrando a necessidade de fortalecer uma rede de vigilância pós-alta capaz de diagnosticar as ISC daqueles pacientes que receberam alta precoce ou não.

De acordo com os resultados obtidos, fica evidente o aumento do tempo de internação requerido para o tratamento da ISC, podendo-se inferir, consequentemente, o aumento dos custos assistenciais envolvidos, além do desgaste do paciente e seus familiares. Nesse sentido, tornase imprescindível o investimento em medidas de prevenção dessas infecções, sendo necessário o envolvimento das equipes assistenciais envolvidas, de profissionais de controle de infecção hospitalar e núcleo de segurança do paciente, para que, juntos, busquem a proposição de melhores práticas assistenciais visando à segurança do paciente e a melhoria da qualidade assistencial. 


\section{REFERÊNCIAS}

1. Souza SF. Estudo do perfil clínico de pacientes adultos submetidos à cirurgia cardíaca pelo sistema único de saúde (SUS) na região metropolitana de Belo Horizonte por meio da aplicação de escore de risco pré-operatório Euroscore [dissertação]. Belo Horizonte (MG): Universidade Federal de Minas Gerais; 2009. 124p. Disponível em: http://www.bibliotecadigital.ufmg.br/dspace/han dle/1843/ECJS-7SDJ93

2. Souza AG, Fichino MAS, Silva GS, Bastos FCC, Piotto RF. Epidemiology of coronary artery bypass grafting at the Hospital Beneficência Portuguesa. Rev Bras Cir Cardiovasc [Internet]. 2015 [citado em 2017 out 20];30(1):33-9. Disponível em: http://www.scielo.br/scielo.php?script=sci arttex t\&pid=S0102-76382015000100009

3. Tarasoutchi F, Momtera MW, Grinberg M, Barbosa MR, Piñeiro DJ, Sánchez CRM, et al. Diretriz Brasileira de Valvopatias - SBC 2011. Arq Bras Cardiol [Internet]. 2011 [citado em 2017 out 20];97(5):11-67. Disponível em: http://publicacoes.cardiol.br/consenso/2011/Dir etriz\%20Valvopatias\%20-\%202011.pdf

4. Magill SS, Hellinger W, Cohen J, Kay R, Bailey C, Boland $B$, et al. Prevalence of healthcareassociated infections in acute care hospitals in Jacksonville, Florida. Infect Control Hosp Epidemiol [Internet]. 2012 [citado em 2017 out 20];33(3):283-91. Disponível em: https://www.ncbi.nlm.nih.gov/pubmed/22314066 5. Agência Nacional de Vigilância Sanitária (Brasil). Medidas de prevenção de infecção relacionada à assistência à saúde. Série Segurança do paciente e qualidade em serviços de saúde. Brasília, DF; 2017. Disponível em: http://portal.anvisa.gov.br/documents/33852/35 07912/Caderno+4+-

+Medidas+de+Preven\%C3\%A7\%C3\%A3o+de+Infe c\%C3\%A7\%C3\%A3o+Relacionada+\%C3\%A0+Assis t\%C3\%AAncia+\%C3\%A0+Sa\%C3\%BAde/a3f23dfb2c54-4e64-881c-fccf9220c373

6. Jenks PJ, Laurent M, McQUuarry S, Watkins R. Cllinical and economic burden of surgical site infection (SSI) and predicted financial consequences of elimination of SSI from an English hospital. J Hosp Infect [Internet]. 2014 [citado em 2017 set 15];86(1):24-33. Disponível em:

http://www.sciencedirect.com/science/article/pii SO195670113003447

7. Kobayashi J, Kusachi S, Sawa Y, Motomura N, Imoto $\mathrm{Y}$, Makuuchi $\mathrm{H}$, et al. Socioeconomic effects of surgical site infection after cardiac surgery in Japan. Surgical Today [Internet]. 2015 [citado em 2017 ago 10];15(4):422-8. Disponível em: https://www.ncbi.nlm.nih.gov/pubmed/24973059

8. Moraes AAI, Abboud CS, Chammas AZL, Aguiar YS, Mendes LC, Farsky PS. Long term mortality of deep sternal wound infection after coronary artery bypass surgery. Rev Bras Cir Cardiovasc [Internet]. 2012 [citado em 2017 nov 10];27(3):377-82. Disponível em: http://www.scielo.br/scielo.php?script=sci arttex t\&pid=S0102-76382012000300007

9. Berti F, Giocomelli S, Amprino V, Pieve G, Ceresetti D, Testa $M$, et al. The "bundle" approach to reduce the surgical site infection rate. J Eval Clin Pract [Internet]. 2017 [citado em 2017 ago 10];23(3):642-7. Disponível em: https://www.ncbi.nlm.nih.gov/pubmed/28145067 10. Araujo MPS, Oliveira AC. Reflexão teórica: quais mudanças poderão ocorrer na assistência cirúrgica após a implantação dos núcleos de segurança do paciente? Rev Enferm Cent O Min [Internet]. 2015 [citado em 2017 ago 10];5(1):1542-51. Disponível em: http://seer.ufsj.edu.br/index.php/recom/article/v iew/807/844

11. Centers for Disease Control and Prevention (CDC). Surveillance definitions for specific types of infections: National Healthcare Safety Netwoek (NHSN) manual, patient safety component protocol. MMWR Recomm Rep. RR16; 2015.

12. Janssen MAS, Azevedo PR, Silva LDC, Dias RS. Perfil sociodemográfico e clínico de pacientes submetidos à cirurgia de revascularização do miocárdio. Rev Pesq Saúde [Internet]. 2015 [citado em 2017 out 20];16(1):29-33. Disponível em:

http://www.periodicoseletronicos.ufma.br/index. php/revistahuufma/article/view/4073/2155

13. Silva QCG, Barbosa MH. Fatores de risco para infecção de sítio cirúrgico em cirurgia cardíaca. Acta Paul Enferm [Internet]. 2015 [citado em 2017 out 20];25(2):89-95. Disponível em: http://www.scielo.br/pdf/ape/v25nspe2/pt 14.pdf 14. Bratzler DW, Dellinger P, Olsen KM, Perl TM, Auwaerter PG, Bolon MK, et al. Clinical practice guidelines for antimicrobial prophylaxis in surgery. Am J Health Syst Pharm [Internet]. 2013 [citado em 2017 set 15];70(3):195-283. Disponível em:

http://www.ajhp.org/content/ajhp/70/3/195.full.pdf 15. Cossin S, Malavaud S, Jarno $P$, Giard $M$, L'Hériteau $F$, Simon $L$, et al. Surgical site infection after valvular or coronary artery bypass surgery: 
2008-2011 French SSI national ISSO - RAISIN surveilhance. J Hosp Infect [Internet]. 2015 [citado em 2017 set 15];91(3):1-6. Disponível em: https://www.ncbi.nlm.nih.gov/pubmed/26321674 16. Centers for Disease Control and Prevention (CDC); National Healthcare Safety Network (NHSN). CDC/NHSN surveillance definitions for specific types of infections. Atlanta; 2017. 29p. Disponível em: https://www.cdc.gov/nhsn/pdfs/pscmanual/17ps cnosinfdef current.pdf

17. Morikane K, Honda H, Yamagishi T, Suzuki S. Differences in risk factores associated with surgical site infections following two types of cardiac surgery in Japanese patients. J Hosp Infect [Internet]. 2015 [citado em 2017 ago 10];90:15$21 . \quad$ Disponível em: https://www.ncbi.nlm.nih.gov/pubmed/25623210 18. Robich MP, Sabik JF, Houghtaling PL, Kelava $\mathrm{M}$, Gordon S, Blackstone EH, et al. Prolonged effect of postoperative infections complications on survival after cardiac surgery. Ann Thorac Surg [Internet]. 2015 [citado em 2017 out 10];99:1591$600 . \quad$ Disponível em: https://www.ncbi.nlm.nih.gov/pubmed/25686669 19. Leaper D, Tanner J, Kiernan M. Surveillance of surgical site infection: more accurate definitions and intensive recording needed. J Hosp Infect [Internet]. 2013 [citado em 2017 out 15];83(2):83-6. Disponível em: https://www.ncbi.nlm.nih.gov/pubmed/23332350 20. Tanner J, Padley W, Kiernan M, Leaper D, Norrie $P$, Baggott $R$. A benchmark too far: findings from a national survey of surgical site infection surveillance. J Hosp Infect [Internet]. 2013 [citado em 2017 out 18];83(2):87-91. Disponível em: https://www.ncbi.nlm.nih.gov/pubmed/23332352 21. Young PY, Khadaroo RG. Surgical site infections. Surg Clin North Am [Internet]. 2014 [citado em 2017 out 15];94(6):1245-64. Disponível em: http://www.sciencedirect.com/science/article/pii $\angle$ S0039610914001431

22. Cowper $P$, DeLong ER, Hannan EL, Muhlbaier $\mathrm{LH}$, Lytle $\mathrm{BL}$, Jones $\mathrm{RH}$, et al. Is early too early?: effect shorter stays after bypass surgery. Ann Thorac Surg [Internet]. 2017 [citado em 2017 out 15];83:100-7. Disponível em: https://www.ncbi.nlm.nih.gov/pubmed/17184638 23. Kusachi S, Kashimura N, Komishi T, Shimizu J, Kusunoki M, Oka M, et al. Length of stay and cost for surgical site infection after abdominal and cardiac surgery in japanese hospitals: multicenter surveilhance. Surg Infect [Internet]. 2012 [citado em 2017 out 12];13(4):257-65. Disponível em:

https://www.ncbi.nlm.nih.gov/pubmed/22871224

24. Kelava M, Robich M, Houghtaling PL, Sabik III JF, Gordon S, Mihaljevic T. Hospitalization before surgery increases risk for postoperative infections. J Thorac Cardiovasc Surg [Internet]. 2014 [citado em 2017 out 11];148(4):1615-21. Disponível em: https://www.ncbi.nlm.nih.gov/pubmed/25260276 25. Phillips J, O'Grady H, Baker E. Prevention of surgical site infections. Surgery [Internet]. 2014 [citado em 2017 out 15];32(9):468-71. Disponível em:

http://www.surgeryjournal.co.uk/article/S02639319(14)00135-5/pdf

Nota: Parte integrante da dissertação intitulada: "Fatores determinantes da infecção de sítio cirúrgico em pacientes submetidos a cirurgias de revascularização do miocárdio e implantes de válvulas cardíacas". Belo Horizonte: Escola de Enfermagem, Universidade Federal de Minas Gerais; 2017.

Recebido em: 26/02/2017

Aprovado em: 26/04/2018

Endereço de correspondência:

Nelma de Jesus Braz

Av. Governador Benedito Valadares, 646 - João Pinheiro CEP: 30530-570 - Belo Horizonte/MG - Brasil

E- mail: nelmadejesusbraz@gmail.com 